\title{
Dental treatment considerations for a pediatric patient with incontinentia pigmenti (Bloch-Sulzberger syndrome)
}

Amy Yi-Ling Chen ${ }^{1}$, Kevin Chen ${ }^{2}$

Correspondence: Dr. Amy Yi-Ling Chen

Email: camy4098@cgmh.org.tw

\begin{abstract}
'Pediatric Dentistry Division, Dental Department, Chang Gung Memorial Hospital, Chang Gung University, Taoyuan, Taiwan,

${ }^{2}$ Medical Department, Medical School, China Medical University, Taichung, Taiwan
\end{abstract}

\section{ABSTRACT}

Incontinentia pigmenti (IP) is a uncommon gene disorder, heritage with X-linked dominant mode. IP patients have a characteristic dentition varying from marked hypodontia to delayed eruption and conical crowns on both dentitions. A 51/2-year-old girl, whose mother and younger sister were also diagnosed with IP, has the whirling-like pigmented skin lesion over her trunk and four extremities. Four primary teeth and multiple permanent tooth germs were found to be congenital missing. Dental considerations of further treatment were discussed with her parents including the preservation of primary molars, possible interim prosthesis in mixed or permanent dentition, full mouth rehabilitation with orthodontic and prosthodontic combined treatment, and implant therapy in adulthood. Early and longitudinal involvement of pediatric dentist to deal with the dental complications of IP can not only solve the esthetic problem and oral function but also maintain the oral health of children with IP to adulthood.

Key words: Congenital missing, dermal pigmented lesion, hypodontia, incontinentia pigmenti

\section{INTRODUCTION}

Incontinentia pigmenti (IP) which was also known as Bloch-Sulzberger syndrome is an uncommon gene disorder, heritage with X-linked dominant mode, and usually lethal prenatally in males. ${ }^{[1]}$ This disease affecting approximately one in 40,000 newborns is a multisystem neuroectodermal disorder predominantly affecting the skin, teeth, and other neuroectodermal origin structures. ${ }^{[2]}$ The diagnosis of IP was mostly based on the clinical feature, especially cutaneous findings that presented at birth or developed soon after birth. Mutations in the nuclear factor $(\mathrm{NF}){ }_{\mathrm{K}} \mathrm{B}$ essential modulator (NEMO) gene, also known as the inhibitor of kappa B kinase gamma

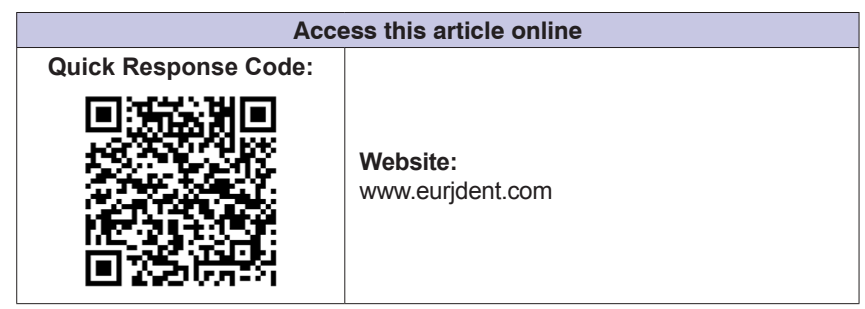

gene which located in the $\mathrm{Xq} 28$ chromosomal region, have been shown to be responsible for IP ${ }^{[1]}$ NEMO gene is required for NF-KB activation pathways that played important roles in inflammation, immune response, cell growth control, and protection against apoptosis. NEMO gene deletion was accounted for $80 \%-90 \%$ of all IP. ${ }^{[1]}$ There is no ethnic or geographic predominance although most cases have been reported in Caucasians.

In the diagnosed IP care, the skin demonstrated swirling patterns of melanin pigmentation, resembling the appearance of marble cake, mostly located over

This is an open access article distributed under the terms of the Creative Commons Attribution-NonCommercial-ShareAlike 3.0 License, which allows others to remix, tweak, and build upon the work non-commercially, as long as the author is credited and the new creations are licensed under the identical terms.

For reprints contact: reprints@medknow.com

How to cite this article: Chen AY, Chen K. Dental treatment considerations for a pediatric patient with incontinentia pigmenti (Bloch-Sulzberger syndrome). Eur J Dent 2017;11:264-7.

DOI: 10.4103/ejd.ejd_95_17 
trunk and extremities. The hyperpigmentation will persist for several years and usually disappears before the age of 20 years. ${ }^{[3]}$ In accordance with Landy and Donnai's criteria, skin lesions were classified as IP major criteria, such as erythema; hyperpigmented streaks and whorls; and pale, hairless, atrophic linear streaks or patches. For dental, hair, nail, and retinal anomalies except in the special condition were classified as IP minor criteria. Minic et al. performed a research on the available published data during 1993-2010. ${ }^{[4]}$ The result showed that $54 \%$ of the investigated IP patients had dental-related anomalies. The most frequent types of dental manifestations were dental shape anomalies and hypodontia. For the oral anomaly types, cleft palate and high-arched palate were found to be more common. ${ }^{[4]}$ However, from other researches about the dental and oral anomalies in different IP cohorts, the prevalence of dental anomalies including marked hypodontia to delayed eruption and conical crowns in both dentitions varied from $30 \%$ to $92 \%$. The purpose of this case report was to set up a possible treatment protocol integrating the comprehensive dental treatments to rehabilitate the function and esthetics and to maintain the dental health of the patient with IP.

\section{CASE REPORT}

A 51/2-year-old female patient was brought to our dental clinic because of lower primary incisors shedding. She had whirling-like pigmented lesions over the trunk and four extremities [Figure 1]. The patient's mother also had similar skin lesions and pigmentations when she was young, but all lesions disappeared in adulthood. Parents reported that she was diagnosed as IP confirmed by a histopathological skin biopsy taken from her legs when she was 6 months old. Familial chromosome study was done and demonstrated that both the girl and her mother had four nucleotides deletion.

This girl had symmetrical facial appearance with straight facial profile [Figure 1]. On intraoral photography, this girl demonstrated that bimaxillary incisors were in the relationship of crossbite with acceptable oral hygiene with healthy gingiva condition [Figure 2]. Bilateral lower primary incisors were shedding, and proximal caries were noted between upper left primary molars. A primary dentition with generalized spacing and four primary teeth congenital missing (\#52, \#54, \#62, \#74) were demonstrated on periapical and bitewing X-rays [Figure 3]. Multiple permanent tooth germs (\#12, \#14, \#15, \#17, \#22,

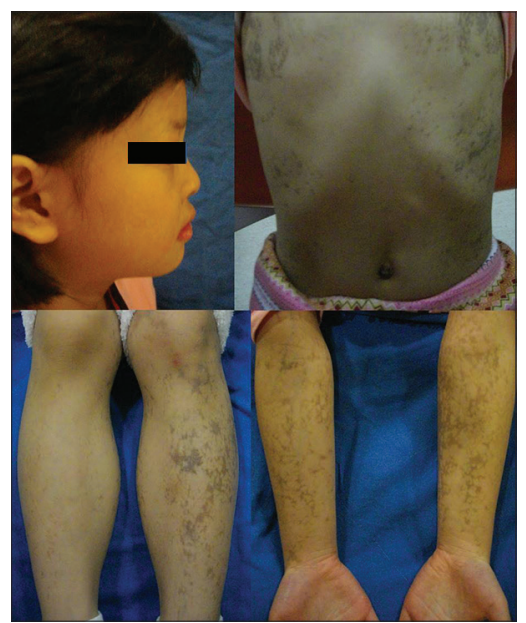

Figure 1: Patient presented a straight lateral profile. Whirling-like pigmented lesions over trunk and four extremities

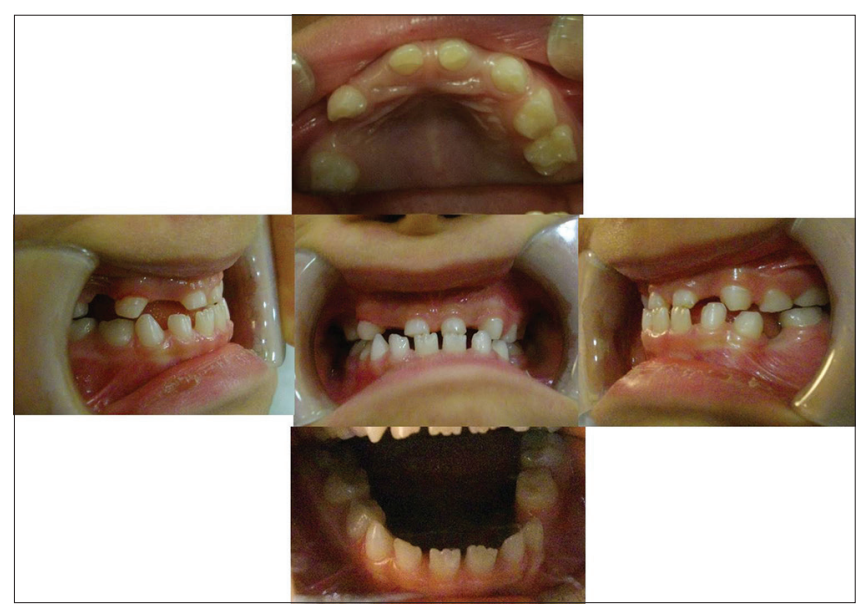

Figure 2: A full serial of intraoral photography at recall visit. Healthy gingiva condition and anterior crossbite with four primary teeth congenital missing

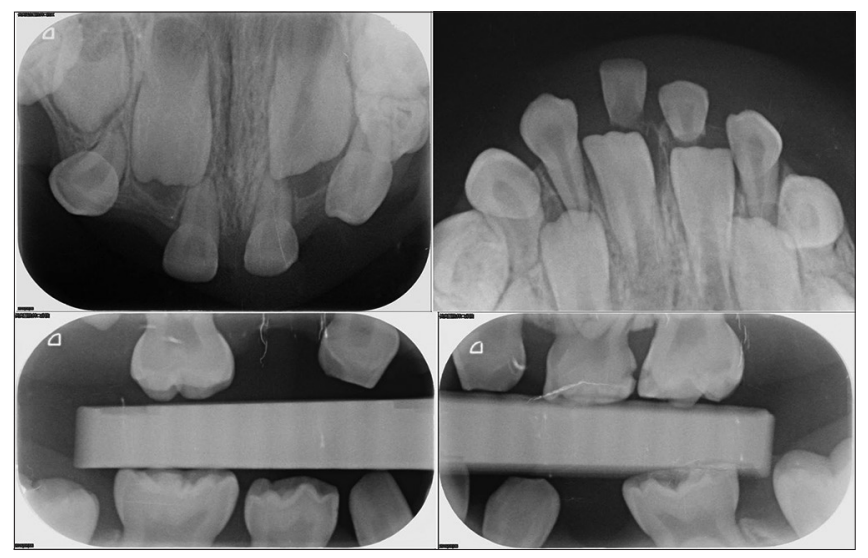

Figure 3: Lower primary incisors shedding with permanent incisors erupting. A primary dentition with generalized spacing with proximal caries between upper left primary molars, four primary teeth (\#52, \#54, \#62, \#74), and bilateral maxillary permanent lateral incisors (\#12, \#22) congenital missing noted

\#25, \#34, \#35, \#37, \#45) congenital missing were also found on the panoramic and periapical X-ray films 


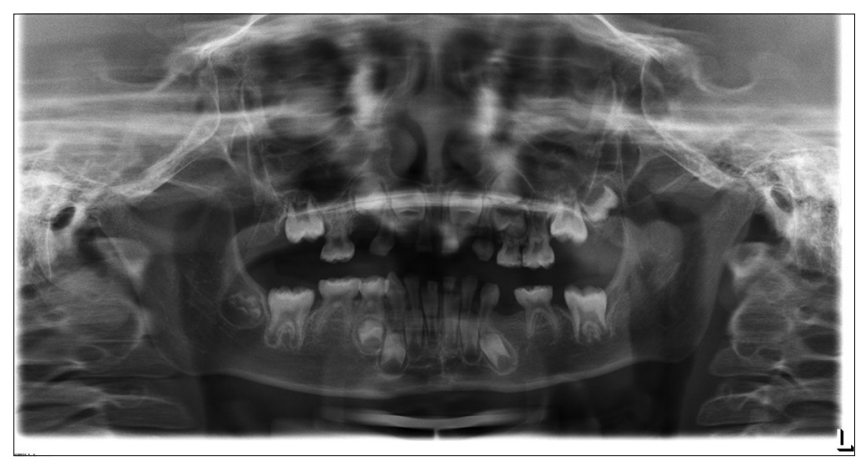

Figure 4: Panoramic $X$-ray showed multiple permanent tooth germs (\#12, \#14, \#15, \#17, \#22, \#25, \#34, \#35, \#37, \#45) and four primary teeth (\#52, \#54, \#62, \#74) congenital missing

[Figures 3 and 4]. The shedding lower primary central incisors were extracted on the day of the first dental visit. Further dental treatments for proximal caries and caries prevention were arranged and performed. Six months after initial dental treatments, the child was brought back for regular preventive dental treatment to maintain the oral health. The parents were informed about the influence of IP on the child's present dental condition, and a thorough consultation was performed with the involvement of her parents about the possible future dental treatment options. First, we suggested the patient to have regular preventive dental treatment for the dental health maintenance every 6 months. Second, because of all four second premolars were congenital missed, four primary second molars will try to be preserved as long as they can to replace the function of second premolars if possible. Third, we will keep follow-up the growth of maxilla and mandible, the change of malocclusion combined with the eruption of permanent teeth in mixed dentition. Fourth, reshaping of malformed teeth and interim denture fabrication will be performed when transitory esthetic rehabilitation needed in mixed dentition or young permanent dentition. Because the skeletal and occlusal relationships might be influenced by growth during puberty and the more appropriate timing for implant therapy was suggested after growth spurt, full mouth orthodontic/prosthodontic combined treatments for tooth relocation and alignment to achieve better result of full mouth rehabilitation will be set up and performed finally in adulthood. A written informed consent was obtained from patient's parents for publication of this case report and accompanying images at the end of visit.

\section{DISCUSSION}

Most of the reports published about IP were from western populations. Only few reports related to
East Asian population have been published including Korea, ${ }^{[5]}$ Japan, ${ }^{[6]}$ China, and Taiwan. ${ }^{[7]}$ From those researches, the frequency of NEMO mutation and the susceptibility to IP of Asian populations are similar to those in Western populations. A previous report from Taiwan, presented that NEMO mutation was detected in $66.7 \%$ of participants with IP, but only three cases of them were familial. This case report presented to be a familial IP with deletion of four genes. Further research can be done to find out the correlation of clinical manifestations between her family members and to clarify which factors might relate to the variation of dental findings.

Landy and Donnai classified IP criteria into two groups. Anomalous dentition was defined as major criteria instead of minor criteria in the group with first-degree family history. ${ }^{[8]}$ However the primary tooth starts to eruption after the age of 6 months old and some cases with IP have the possible complication of delayed eruption. Therefore, dental anomalies were difficult to be used as diagnosis tool for the children younger than 1 year old. This case reported even with the impression of familial IP combined with clinical dermatologic manifestations at 4 months old, the confirmation of definitive diagnosis was needed and obtained by a histopathological examination. In addition, chromosomal study was done to detect the location of genetic deletion and to make sure whether involving any other gene mutations since NEMO gene rearrangement is not the only gene mutation related to IP.

Tooth agenesis, also known as hypodontia, was the most frequent dental anomaly in IP. The reported incidences of hypodontia were $2.7 \%-12.2 \%$ in the permanent dentition of normal population. ${ }^{[9]}$ However, the prevalence of tooth agenesis in the analyzed IP was $31.2 \%$ that was much higher than the prevalence in different ethnic groups, both males and females. ${ }^{[4]}$ In the maxillary arch, the most common missing teeth were the lateral incisors followed by the second premolars with the incidence of $87.7 \%$ and $10.4 \%$, respectively. In the mandibular arch, the most common missing teeth were the second premolar with a high incidence of $64.3 \%{ }^{[9]}$ All of the mentioned teeth were missing congenitally occurs within this report. The most common dental finding associated with hypodontia was the prolong retention of deciduous molars. The retained mandibular deciduous second molar was reported with a long life span. ${ }^{[10,11]}$ This phenomenon can provide us a good interim treatment option to handle the possible complication caused by the premolar 
congenital missing in young permanent dentition until adulthood then we can perform a full mouth definitive rehabilitation. Therefore, we can try to preserve four second primary molars for replacing the congenital missed second premolars instead of the interim denture fabrication to maintain the premolar's space that will be rehabilitated by implant therapy in adulthood. Treatment of children with several congenitally missing teeth is challenging, especially combined with malocclusion, because growth and development of the oral structure must be considered at the same time. The treatment plan for the patient with missing teeth should be based on a comprehensive evaluation of the patient's age, occlusal and masticatory function, requirements for tooth reshaping and tooth alignment, and the possible need of esthetic consideration. Dental supervision and management would more focus on the cosmetic correction of the teeth malformation and dental health during the growth period. Final definitive treatment planning should be set up after growth spurt to have a better prognosis and esthetic result with minimal tooth structure preparation. The treatment protocol of this case report was based on above clinical findings and reasons.

\section{CONCLUSION}

Dental and oral anomalies of the patient with IP were seldom causing life-threatening problem. However, some anomalies might cause feeding problems, for example, cleft lip and cleft palate. The hypodontia could cause serious psychological problems for IP patient with visible signs of congenital anomaly by influencing the patient's esthetics and the quality of life. With the longitudinal involvement and cooperation of different dental specialties and the proper treatment protocol, the child with IP still can obtain good dental esthetics and functional rehabilitation.

\section{Declaration of patient consent}

The authors certify that they have obtained all appropriate patient consent forms. In the form the patient(s) has/have given his/her/their consent for his/her/their images and other clinical information to be reported in the journal. The patients understand that their names and initials will not be published and due efforts will be made to conceal their identity, but anonymity cannot be guaranteed.

\section{Financial support and sponsorship \\ Nil.}

\section{Conflicts of interest}

There are no conflicts of interest.

\section{REFERENCES}

1. Smahi A, Courtois G, Vabres P, Yamaoka S, Heuertz S, Munnich A, et al. Genomic rearrangement in NEMO impairs NF-kappaB activation and is a cause of incontinentia pigmenti. The International incontinentia pigmenti (IP) Consortium. Nature 2000;405:466-72.

2. Ehrenreich M, Tarlow MM, Godlewska-Janusz E, Schwartz RA Incontinentia pigmenti (Bloch-Sulzberger syndrome): A systemic disorder. Cutis 2007;79:355-62.

3. Landy SJ, Donnai D. Incontinentia pigmenti (Bloch-Sulzberger syndrome). J Med Genet 1993;30:53-9.

4. Minic S, Trpinac D, Gabriel H, Gencik M, Obradovic M. Dental and oral anomalies in incontinentia pigmenti: A systematic review. Clin Oral Investig 2013;17:1-8.

5. Kim BJ, Shin HS, Won CH, Lee JH, Kim KH, Kim MN, et al. Incontinentia pigmenti: Clinical observation of 40 Korean cases. J Korean Med Sci 2006;21:474-7.

6. Okita M, Nakanishi G, Fujimoto N, Shiomi M, Yamada T, Wataya-Kaneda M, et al. NEMO gene rearrangement (exon 4-10 deletion) and genotype-phenotype relation in Japanese patient with incontinentia pigmenti and review of published work in Japanese patients. J Dermatol 2013;40:272-6.

7. Hsiao PF, Lin SP, Chiang SS, Wu YH, Chen HC, Lin YC. NEMO gene mutations in Chinese patients with incontinentia pigmenti. J Formos Med Assoc 2010;109:192-200.

8. Hadj-Rabia S, Froidevaux D, Bodak N, Hamel-Teillac D, Smahi A, Touil Y, et al. Clinical study of 40 cases of incontinentia pigmenti. Arch Dermatol 2003;139:1163-70.

9. Al-Abdallah M, AlHadidi A, Hammad M, Al-Ahmad H, Saleh R. Prevalence and distribution of dental anomalies: A comparison between maxillary and mandibular tooth agenesis. Am J Orthod Dentofacial Orthop 2015;148:793-8.

10. Bjerklin K, Al-Najjar M, Kårestedt H, Andrén A. Agenesis of mandibular second premolars with retained primary molars: A longitudinal radiographic study of 99 subjects from 12 years of age to adulthood. Eur J Orthod 2008;30:254-61.

11. Park JH, Tai K, Yuasa K, Hayashi D. Multiple congenitally missing teeth treated with autotransplantation and orthodontics. Am J Orthod Dentofacial Orthop 2012;141:641-51. 